\title{
'||-||||||||||||||||||||||||||||||||||||||||||||||||||||||||||||||||||||.
}

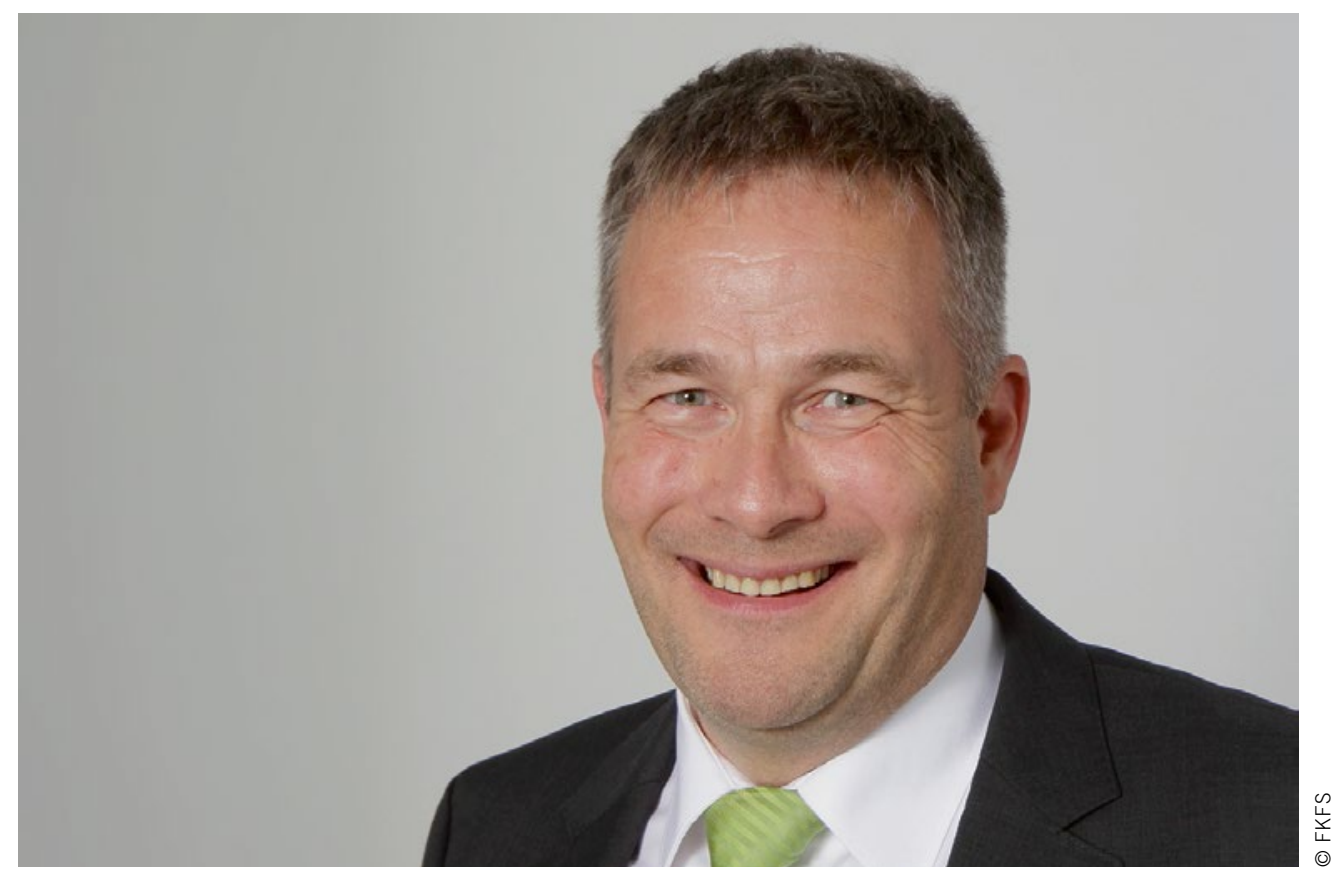

Prof. Dr.-Ing.

Hans-Christian Reuss

Chair of Automotive Mechatronics,

University of Stuttgart (Germany)

\section{New Statics for Onboard Architectures}

All indications are that the vehicle of the future will be electric, driverless and interconnected. But what will this mean exactly in terms of the technological developments that will be necessary to make it so? After all, the sticker price is climbing just as quickly as the complexity of the relevant electronic, software and IT solutions. The challenges have been formulated and we can expect that suitable proposals will follow, proposals, for instance, that are based on new and sustainable system architectures and that also take account of growing concerns relating to safety and security. But is this the development that we are actually seeing?

These issues need to be examined. In my opinion, it will not suffice to simply rely on the standard evolutionary approach to systems development. But that is what is happening. Unreflectively, we have already come to depend on distributed, parallel computing systems. Five different data buses are standardly used to enable communication between up to 100 control devices. One gateway node handles the communication between the buses and serves as a diagnostic interface to the outside world. Initial applications make use of Ethernet technology. The solution is simply to expand the architecture as new functionalities are added - another balcony here and another dormer there. At some point, this will begin to come at the cost of structural integrity.

It would be better to form domains - with a few high-capacity control units that are connected to one another via a backbone. The functionalities can then be compiled, optimised and outfitted with more computing capacity according to domain.
Both the car-to-X links and over-the-air software updating functions could be simplified. This would create space for new design and security methods.

A new E/E architecture will make use of central supercomputers. In addition to the above-mentioned advantages, we would be able to develop completely new software architectures and, in combination with increased wireless capacity, we could outsource functionalities to cloud systems. This would also open vehicles up to open-software platforms and machinelearning features. Hypervisor concepts could then be realised. It would no longer be the hardware, but the software that would describe the system.

The skeptics may hasten to point out that the necessary resources just are not available and that it would also be necessary to restructure development departments - and all for something that customers would not notice anyhow. Or would they? Interestingly enough, a number of companies in the United States and China do not feel so bound to the retention of 30 -year-old architectures. They have the advantage of starting out without the baggage - a competitive advantage that the customers will indeed notice sooner or later.

At least there are a few joint projects of a revolutionary as opposed to an evolutionary - nature being carried out by the classic automobile manufacturers. And the software industry has introduced some revolutionary concepts for controlsystem architecture. I just wish there were many more such efforts underway. 